\title{
HYPOSULPHITE OF SODA IN INFLAMMATORY RHEUMATISM.
}

[Communicated for the Boston Bedical and Surgical Journal.]

'THIs preparation of soda is worthy of more notice as a remedial agent than is usually accorded to it by the profession. The following case will illustrate the value of the remedy.

Mr. C. had been ill for three days with inflammatory rheumatism when I first visited him. He had chills once in four hours. Tongue dry, with a dark, thick coat. Pulse 120 , dicrotic; shin very dry; urine high colored and scanty, with brickdust sediment. All the joints very much swollen, inflamed and exceedingly painful. The pain was so severe that the walking of any one upon the carpet caused him to complain most bitterly. Ordered the following :-R. Hyd. chlor. mitis, gr. viij.; pulvi. jalap., gr. xx. ; pulv. digitalis, gr. $x$. Divide into two powders, one to be taken once in three hours; followed by R. Nitrate potassa, gr. Xv. ; syrupi simplicis, $\int_{3}$ ij. ; vin colchici sem., 3 ij. ; morphiæ, gr. ij. M. Sumat cochleare medium quarter horis. Externally apply R. 'Ir. aconite, gtt. xl. ; acrt. plumbi, 3iij. ; tr. opii, \{3j.; alcohol, z vij. M. Bathe the parts with this mixlure freely, applying cotton after each application.

$2 \mathrm{~d}$ day.--ls no better ; skin dry ; knees, which are mosily complained of, very painful and badly swollen. Vomited twice through the night. Pulse 140, full. Passes urine more freely. Superadded pericardial inflammation. Cannot be moved. Countenance anxious. Breathing quick. Pain and some oppression about the cardiac region. R. Hyposulphite sodæ, $3 \mathrm{j}$. ; acet. morphiæ, gr. ij.; gum acacie, gr. xx. M. Divide into six powders, one to be taken every two hours. Apply, externally. cloths wet in R. Hyposulphite sodæ, $z_{j}$. ; acid. acet. dil., Oss. M. Renewed once in three hours.

3d day.-Had a good night's rest. Tongue looks betrer; pulse 100 ; skin moist ; urine free, not very high colored ; swelling much diminished. Has but little pain. At his request let him sit up. Continue the same medicine.

4 th day.-Pulse 88 ; swelling nearly gone; no pain; sits up. The same medicine continued.

5 th day.-Tongue clean; pulse 80 ; walks about the room. Has some appetite. Give him chicken broth. Medicine continued. Two comp. calh. pills at night.

6th day.-No pain. Pulse 78. At his request let him go out. Medicine continued, with a little brandy and water.

8th day.—Discharged, .well.

The fact most worthy of notice is the wonderful power of the medicine in exciting the secretion of the kidneys, when internally used. As an antiphlogistic agent, I have found it very powerful, and have used it externally where lead was objectionable, and some other agents of this class of remedies were useless.

Newoton Upper Falls, Feb. 26th, 1855.

J. H. W ARREN. 